\title{
Optimal cerebral perfusion pressure via transcranial Doppler in TBI: application of robotic technology
}

\author{
Frederick A. Zeiler ${ }^{1,2,3}$ (D) Marek Czosnyka ${ }^{4,5} \cdot$ Peter Smielewski $^{4}$
}

Received: 29 July 2018 / Accepted: 20 September 2018 / Published online: 29 September 2018

(C) The Author(s) 2018

\begin{abstract}
Individualized cerebral perfusion pressure (CPP) targets may be derived via assessing the minimum of the parabolic relationship between an index of cerebrovascular reactivity and CPP. This minimum is termed the optimal CPP (CPPopt), and literature suggests that the further away CPP is from CPPopt, the worse is clinical outcome in adult traumatic brain injury (TBI). Typically, CPPopt estimation is based on intracranial pressure (ICP)-derived cerebrovascular reactivity indices, given ICP is commonly measured and provides continuous long duration data streams. The goal of this study is to describe for the first time the application of robotic transcranial Doppler (TCD) and the feasibility of determining CPPopt based on TCD autoregulation indices.
\end{abstract}

Keywords CPP optimum $\cdot$ Robotic transcranial Doppler $\cdot$ TCD $\cdot$ Traumatic brain injury $\cdot$ TBI

\section{Introduction}

Continuous monitoring of cerebrovascular reactivity in traumatic brain injury (TBI) is becoming increasingly common in the multi-modal monitoring (MMM) of critically ill patients $[10,11,16]$. To date, support for such monitoring has arisen within international consensus statements $[10,16]$. Such support is centered on what is considered the "gold standard" continuous index, pressure reactivity index (PRx — correlation between intracranial pressure (ICP) and mean arterial pressure (MAP)) [11], given its association with global outcome in TBI and validation in experimental animal models against the

This article is part of the Topical Collection on Brain trauma

Electronic supplementary material The online version of this article (https://doi.org/10.1007/s00701-018-3687-5) contains supplementary material, which is available to authorized users.

Frederick A. Zeiler

umzeiler@myumanitoba.ca

Marek Czosnyka

mc141@medschl.cam.ac.uk

Peter Smielewski

ps10011@cam.ac.uk

1 Division of Anaesthesia, Addenbrooke's Hospital, University of Cambridge, Cambridge, UK lower limit of autoregulation [4, 23, 32]. Furthermore, literature supports the association of PRx-derived "personalized" cerebral perfusion pressure (CPP) targets (referred to as CPP optimum or CPPopt) and global outcome [19].

Numerous other continuous indices of cerebrovascular reactivity exist in the TBI literature [31, 32], derived from other invasive and non-invasive monitoring devices, including transcranial Doppler (TCD)-based measures. Indices based on TCD mean flow velocity (FVm) and systolic flow velocity (FVs) have been linked to global outcome in adult TBI $[6,22]$ and are known to be reasonably strongly associated with ICPderived indices, such as PRx $[30,33]$.
2 Department of Surgery, Rady Faculty of Health Sciences, University of Manitoba, Winnipeg, MB, Canada

3 Clinician Investigator Program, Rady Faculty of Health Sciences, University of Manitoba, Winnipeg, MB, Canada

4 Division of Neurosurgery, Department of Clinical Neurosciences, Addenbrooke's Hospital, University of Cambridge, Cambridge CB2 OQQ, UK

5 Institute of Electronic Systems, Warsaw University of Technology, Warsaw, Poland 
However, despite the success with CPPopt determination using ICP indices [1, 19], it is unknown if TCD-based measures can be used to estimate this value, despite the strong association with ICP indices. This has not been explored in the past given TCD is typically limited by intermittent short duration recordings, given technical limitations. Various devices and probe holders have been designed for attempts at longer duration recordings, even with automated flow velocity detection algorithms [18, 28]. There are two main methods for assessment of cerebral physiology/structure that have been developed, including automated/semi-automated TCD methods (including robotic TCD) and phased-arrayfocused duplex methods $[3,20]$. Such devices include but are not limited to those developed and/or distributed by Compumedics (Compumedics Germany GmbH, Sigen, Germany, https://www.compumedics.com.au/diagnosticsolution/transcranial-doppler/), Delica (Delica, Shenzhen, China, http://www.delicasz.com/html/en), and Pulse Medical (Pulse Medical Limited, Knaphill, UK, www.pulsemedical. co.uk/). However, with advancement in robotic TCD technology, it is possible to obtain relatively uninterrupted, extended duration recordings, allowing for the ability to assess CPPopt. In this article, we present a descriptive analysis of the first attempts at estimating CPPopt in critically ill adult TBI patients using extended duration recordings obtained from robotic TCD.

\section{Methods}

\section{Patient population}

This was a prospective observational study conducted over a 6-month period within our unit, during which we obtained a robotic TCD unit on trial. All patients suffered from moderate to severe TBI and were admitted to the neurosciences critical care unit (NCCU) at Addenbrooke's Hospital, Cambridge, during the period of November 2017 to May 2018. Patients were intubated and sedated given the severity of their TBI. Invasive ICP monitoring was conducted in accordance with the Brain Trauma Foundation (BTF) guidelines. Therapeutic measures were directed at maintaining ICP less than $20 \mathrm{mmHg}$ and CPP greater than $60 \mathrm{mmHg}$.

TCD is a part of standard intermittent cerebral monitoring within the NCCU. The application of the newer robotic TCD device were therefore in alignment with our usual care, negating the need for formal direct or proxy consent. All data related to patient admission demographics and high frequency digital signals from monitoring devices were collected in an entirely anonymous format, negating the need for formal consent, as in accordance with institutional research committee policies. Given limitations with the device, as outlined in a previous publication [34], not all patients admitted to the NCCU with
TBI could be recorded with this device. In particular, the presence of decompressive craniectomy or unstable cervical spines precluded the application of the TCD device. In general, given the limitations outlined in our previous technology analysis [34], this led to our ability to apply the device in approximately $60-70 \%$ of all TBI patients admitted during the above described time period, with the majority being excluded secondary to decompressive craniectomy (primary or secondary) and having uncertain status of cervical spine stability. No patients were excluded after data sampling with the TCD device.

\section{Signal acquisition}

Various signals were obtained through a combination of invasive and non-invasive methods. Arterial blood pressure (ABP) was obtained through either radial or femoral arterial lines connected to pressure transducers (Baxter Healthcare Corp. CardioVascular Group, Irvine, CA). ICP was acquired via an intra-parenchymal strain gauge probe (Codman ICP MicroSensor; Codman \& Shurtleff Inc., Raynham, MA). Zeroing of the arterial line occurred at the level of the tragus during the course of this study.

Finally, TCD assessment of MCA CBFV was conducted via a robotic TCD system, the Delica EMS 9D (Delica, Shenzhen, China, http://www.delicasz.com/html/en). This system allows for continuous extended duration recording of MCA CBFV, using 1.6 MHz robotically controlled TCD probes, with automated correction algorithms for probe shift. We aimed to record 3 to $4 \mathrm{~h}$ of continuous data from all devices simultaneously, given the previous work from our group on inter-index relationships focused on recording durations of only 0.5 - to 1 -h duration due to limitations of conventional TCD $[22,30]$. Based on manufacturer specifications of the Delica robotic TCD system, the thermal index for the 1 . $6 \mathrm{MHz}$ Doppler probes is less than 1.0, with the index less than 0.5 in most cases. As such, in keeping with the guidelines for adult TCD provided by the British Medical Ultrasound Society, the thermal index for the device is in the range acceptable for potentially "unlimited" TCD duration, while adhering to the principles of "as low as reasonably achievable" (ALARA) $[5,12,13]$. Thus, there were no concerns with tissue heating as a result of the extended duration recordings using this system. Brain temperature, local or global, was not recorded in this patient cohort. Figure 1 displays the robotic TCD device and set up for recording in critical ill TBI patients.

\section{Signal processing}

Signals were recorded using digital data transfer, with sampling frequency of $100 \mathrm{~Hz}$, using ICM+ software (Cambridge Enterprise Ltd., Cambridge, UK, http://icmplus.neurosurg. cam.ac.uk). Signal artifact was removed using a combination of manual and semi-automated methods within $\mathrm{ICM}+$ prior to further processing or analysis. 
Fig. 1 Example of recording set up for ICP and robotic TCD. ICP intracranial pressure, TCD transcranial Doppler. a Displays Delica robotic TCD headframe with two robotic drives mounted to a ratcheting headband system. b Displays close up image of TCD probe encased in robotic drive. $\mathbf{c}$ Example of set up for multi-modal monitoring with left frontal triple bolt (ICP, microdialysis and brain tissue oxygen monitoring), bifrontal near infrared spectroscopy, robotic TCD. d Delica EMS 9D TCD program display, showing automatic flow velocity sampling algorithm, producing a square grid of sampled insonation positions. The system insonates at multiple sites, finding the area with superior signal quality)

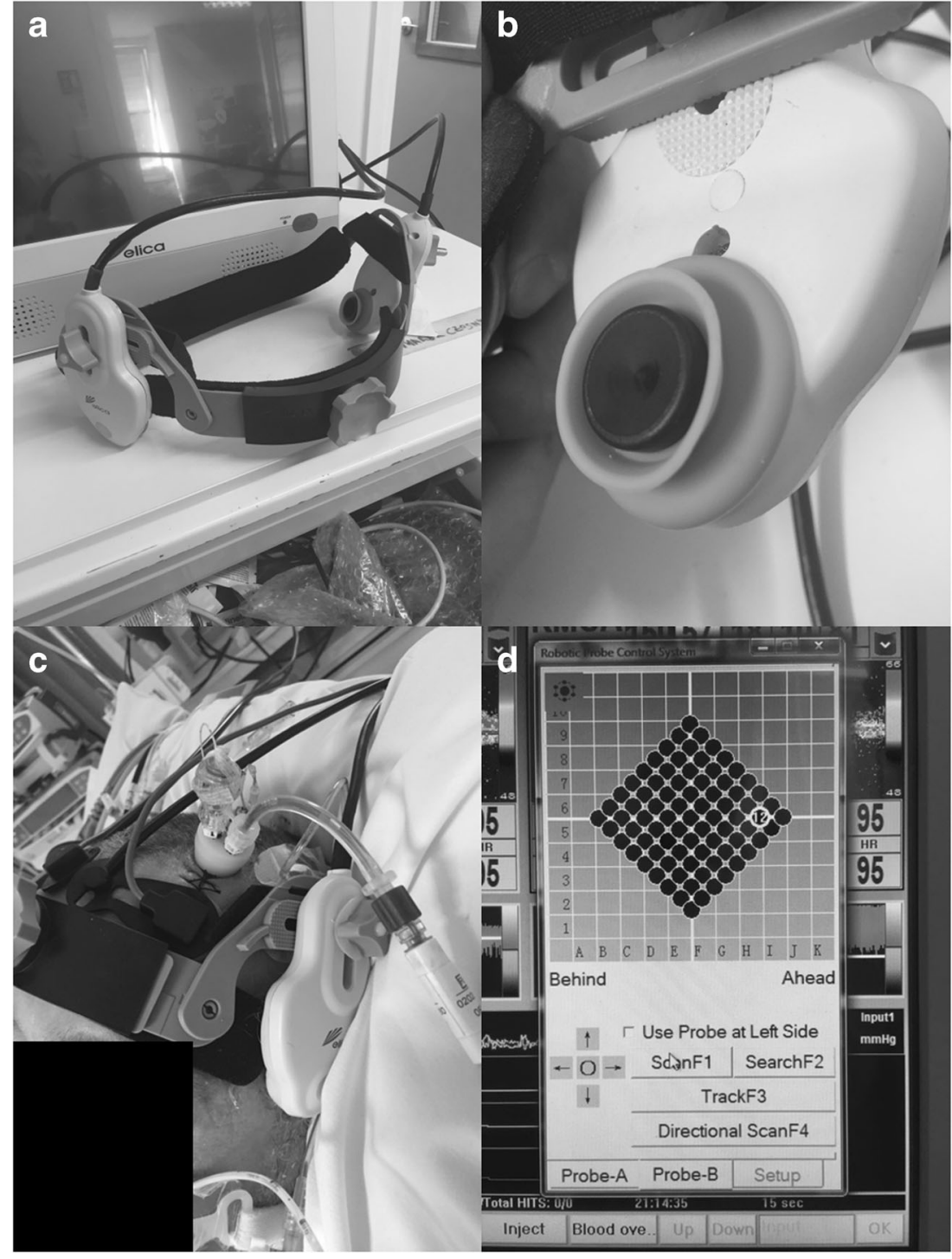

Post-acquisition processing of the above signals was conducted using ICM+ software. CPP was determined using the formula: $\mathrm{CPP}=\mathrm{MAP}-\mathrm{ICP}$. TCD signal was analyzed from the right side in the majority of the patients given right frontal placement of ICP monitors. Therefore, all continuous indices derived are based on mainly right-sided TCD recording. The only exception to this is when we were unable to obtain sufficient quality TCD signal on the right.

Systolic flow velocity (FVs) was determined by calculating the maximum flow velocity (FV) over a $1.5 \mathrm{~s}$ window, updated every second. Diastolic flow velocity (FVd) was calculated using the minimum FV over a $1.5 \mathrm{~s}$ window, updated every second. Mean flow velocity (FVm) was calculated using average FV over a $10 \mathrm{~s}$ window, updated every $10 \mathrm{~s}$ (i.e., without data overlap). Pulse amplitude of ICP (AMP) was determined by calculating the fundamental Fourier amplitude of the ICP pulse waveforms over a $10 \mathrm{~s}$ window, updated every $10 \mathrm{~s}$.
Ten second moving averages (updated every $10 \mathrm{~s}$ to avoid data overlap) were calculated for all recorded signals: ICP, ABP (which produced MAP), CPP, FVm, FVs, andFVd. These non-overlapping 10-s moving average values allow focus on slow-wave fluctuations in signals by decimating the signal frequency to $\sim 0.1 \mathrm{~Hz}$.

Autoregulation indices were derived in a similar fashion across modalities; an example is provided for PRx: A moving Pearson correlation coefficient was calculated between ICP and MAP using 30 consecutive $10 \mathrm{~s}$ windows (i.e., five minutes of data), updated every minute. Details on each index calculation can be found in Table 1.

Data for this analysis were provided in the form of a minute by minute time trends of the parameters of interest for each patient. This was extracted from ICM+ in to comma separated values (CSV) datasets, which were collated into one continuous data sheet (compiled from all patients). 
Table 1 Autoregulation indices and calculation methods

\begin{tabular}{lllll}
\hline Index & Signals correlated & $\begin{array}{l}\text { Signal averaging } \\
(\mathrm{sec})\end{array}$ & $\begin{array}{l}\text { Pearson correlation coefficient } \\
\text { calculation window (min) }\end{array}$ & $\begin{array}{l}\text { Index calculation update } \\
\text { frequency (sec) }\end{array}$ \\
\hline PRx & ICP and MAP & 10 & 5 & 60 \\
PAx & AMP and MAP & 10 & 5 & 60 \\
RAC & AMP and CPP & 10 & 5 & 60 \\
Mx & FVm and CPP & 10 & 5 & 60 \\
Mx_a & FVm and MAP & 10 & 5 & 60 \\
Sx & FVs and CPP & 10 & 5 & 60 \\
Sx_a & FVs and MAP & 10 & 5 & 60 \\
\hline
\end{tabular}

$A M P$, pulse amplitude of ICP; $C P P$, cerebral perfusion pressure; $F V d$, diastolic flow velocity; $F V m$, mean flow velocity; $F V s$, systolic flow velocity; $I C P$, intracranial pressure; $M A P$, mean arterial pressure, min, minute; sec, seconds; $M x$, mean flow index (correlation between FVm and CPP); $M x \_a$, Mx based on MAP (correlation between FVm and MAP); PAx, pulse amplitude index (correlation between AMP and MAP); PRx, pressure reactivity index (correlation between ICP and MAP); $S x$, systolic flow index (correlation between FVs and CPP); Sx_a, Sx based on MAP (correlation between FVs and MAP); RAC, correlation (R) between AMP (A) and $\mathrm{CPP}(\mathrm{C})$

\section{Descriptive analysis}

R statistical software (R Core Team (2016). R: A language and environment for statistical computing. R Foundation for Statistical Computing, Vienna, Austria. URL https://www.Rproject.org/) was utilized for post processing of ICM+ data outputs, producing population wide binned error bar plots of various cerebrovascular reactivity indices versus $\mathrm{CPP}$, to highlight the population-based parabolic relationships between both ICP and TCD indices with CPP. Mean index values were calculated across $5 \mathrm{mmHg}$ bins of CPP for the entire population.

Finally, ICM+ was used to produce individual patient CPPopt plots for the purpose of examining feasibility of TCD-based CPPopt estimation in patients with extended duration uninterrupted recordings (i.e., $\sim 4$-h duration).

\section{Results}

\section{Patient demographics}

During the 6-month trial period for the robotic TCD device, we were able to record 20 critically ill adult TBI patients. Due to limitations imposed by the robotic probe design there are certain contra-indications that prevented its application in some critically ill TBI patients. These include decompressive craniectomy, extensive soft tissue damage to the scalp, and unstable cervical spine (including those with uncertain cervical spine status). Patient demographics can be seen in Appendix A. Overall, the mean age was $42.6 \pm 17.6$ years, with 12 patients being male, and a median admission Glasgow Coma Scale score of 7 (inter-quartile range (IQR): 5 to 8). The mean duration of TCD recording was $224.8 \pm 40.2 \mathrm{~min}$. Though it is acknowledged, not all recordings were completely uninterrupted, given the need for urgent scans and bedside nursing requests for probe removal in 10 patients.

\section{CPP optimum—patient example}

In five patients with the longest duration of uninterrupted recordings (i.e., those reaching $\sim 4 \mathrm{~h}$ in duration), it was possible to estimate CPPopt via plotting mean cerebrovascular reactivity index versus CPP. Figure 2 displays an example of one such recording, where CPPopt could be estimated using both ICP indices (i.e., PRx, PAx, and RAC, see Table 1 for calculations) as well as TCD indices ( $\mathrm{Sx}$ and $\mathrm{Mx}$, see Table 1 for calculations), using $2.5 \mathrm{mmHg}$ bins of CPP. Similar CPPopt plots for the MAP-based Sx_a and Mx_a (see Table 1 for calculations) can be found in Appendix B.

\section{Population wide error Bar plots}

Using the entire 20 patients recorded, we produced various binned error bar plots of ICP and TCD cerebrovascular reactivity indices across $5 \mathrm{mmHg}$ bins of CPP. Figure 3 displays the error bar plots for the ICP-based indices, while Fig. 4 displays the error bar plots for the TCD-based indices. As seen from the plots, at the population level, the TCD-derived indices display a parabolic relationship with CPP, suggesting the feasibility of CPPopt determinations using these TCD measures.

\section{Discussion}

Our simple descriptive analysis of CPPopt estimation using extended duration TCD recordings obtained from robotic TCD highlights what is possible with advanced TCD 

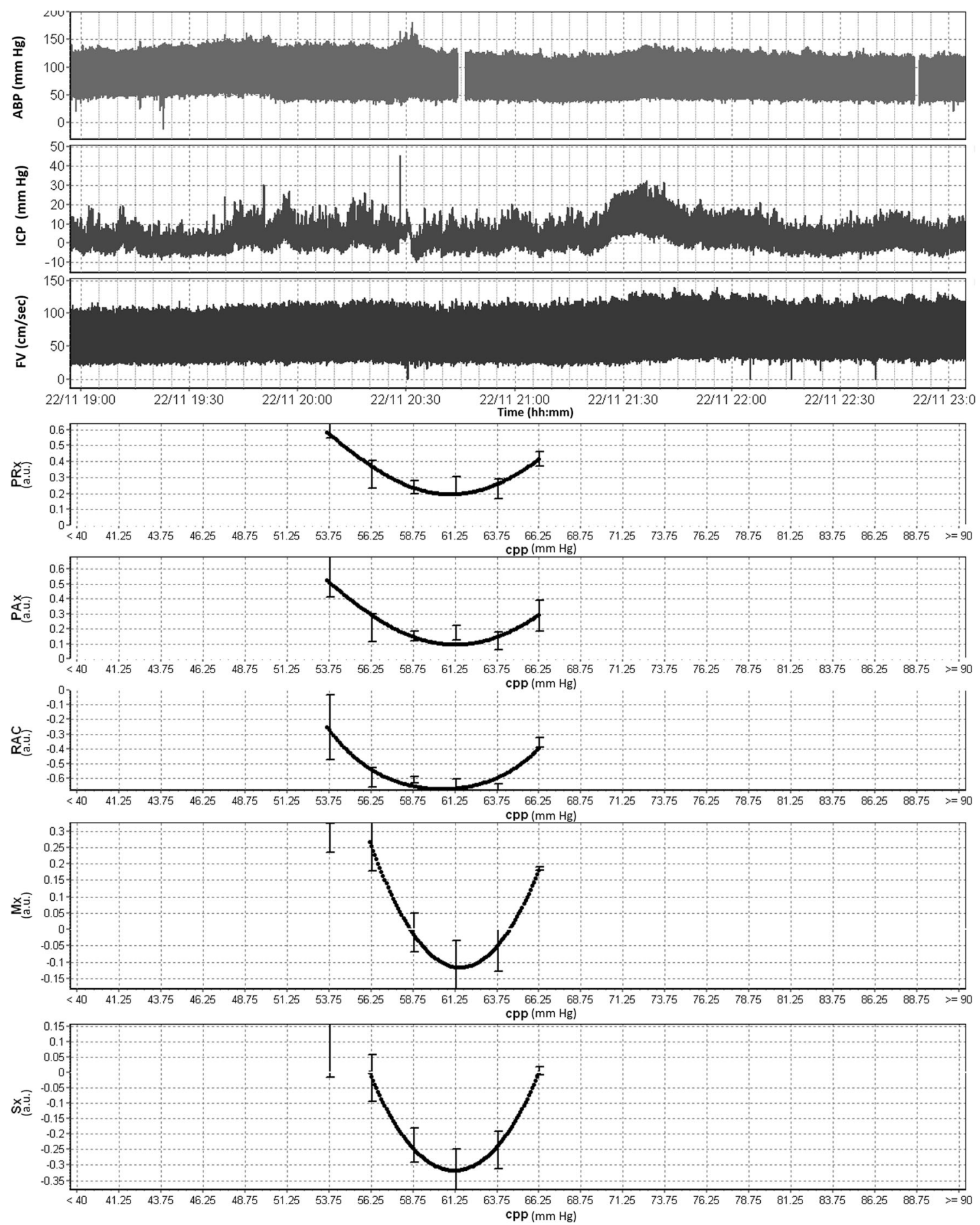

Fig. 2 Patient example of CPPopt estimation using ICP- and TCD-based indices. ABP, arterial blood pressure; AMP, pulse amplitude of ICP; a.u., arbitrary units; cm, centimeters; CPP, cerebral perfusion pressure; FV, flow velocity; FVm, mean flow velocity; FVs, systolic flow velocity; hh:mm, hours:minutes; ICP, intracranial pressure; MAP, mean arterial pressure; $\mathrm{mmHg}$, millimeters of mercury; $\mathrm{Mx}$, mean flow index (correlation between FVm and CPP); PAx, pulse amplitude index (correlation

technology when applied to monitoring critically ill TBI patients. We have demonstrated the feasibility of TCD-based CPPopt estimation, and that these TCD-based cerebrovascular between AMP and MAP); PRx, pressure reactivity index (correlation between ICP and MAP); RAC, correlation between AMP and CPP; sec, second; Sx, systolic flow index (correlation between FVs and $\mathrm{CPP}$ ). *Top 3 panels display raw signal for ABP, ICP and FV over $4 \mathrm{~h}$ window, demonstrating stable continuous TCD recordings. The bottom 5 panels display CPPopt plots for PRx, $\mathrm{PAx}, \mathrm{RAC}, \mathrm{Mx}$, and $\mathrm{Sx}$

reactivity indices do indeed have a parabolic relationship with $\mathrm{CPP}$, as seen classically with ICP-based indices [1, 2, 14, 17], though it must be acknowledged that the duration of 
a

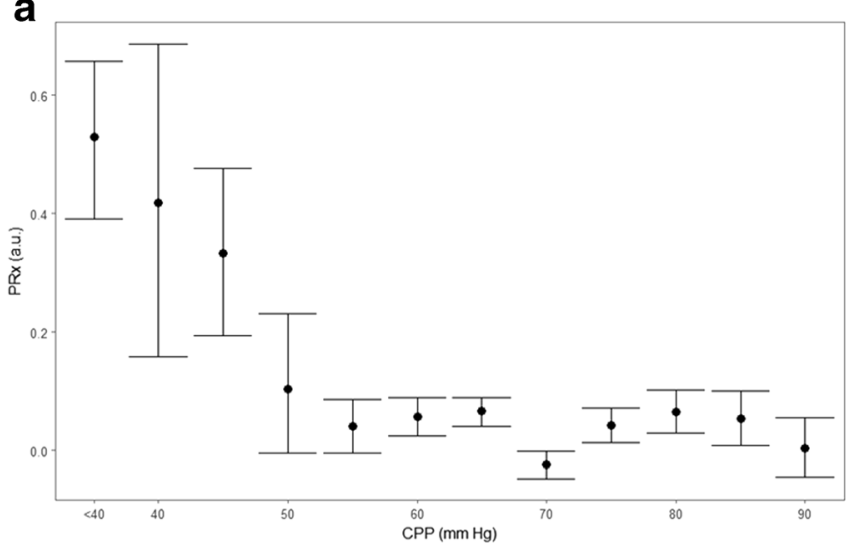

b

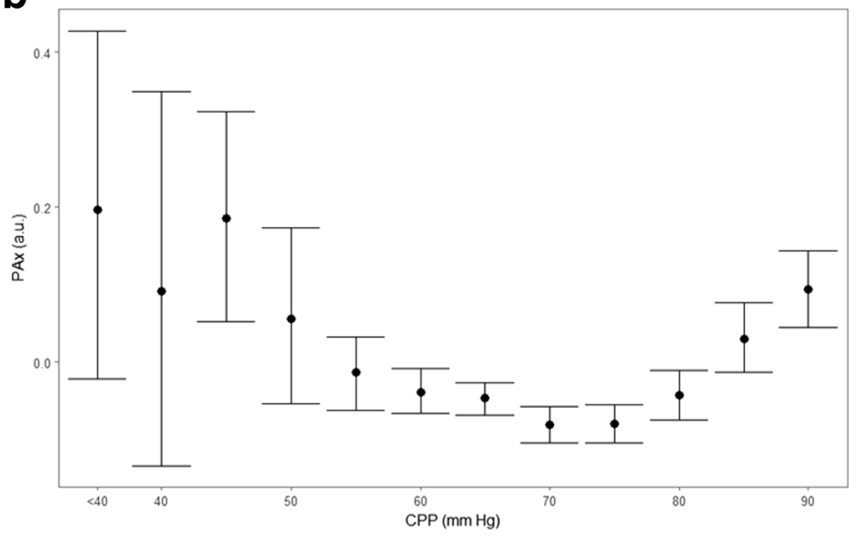

C

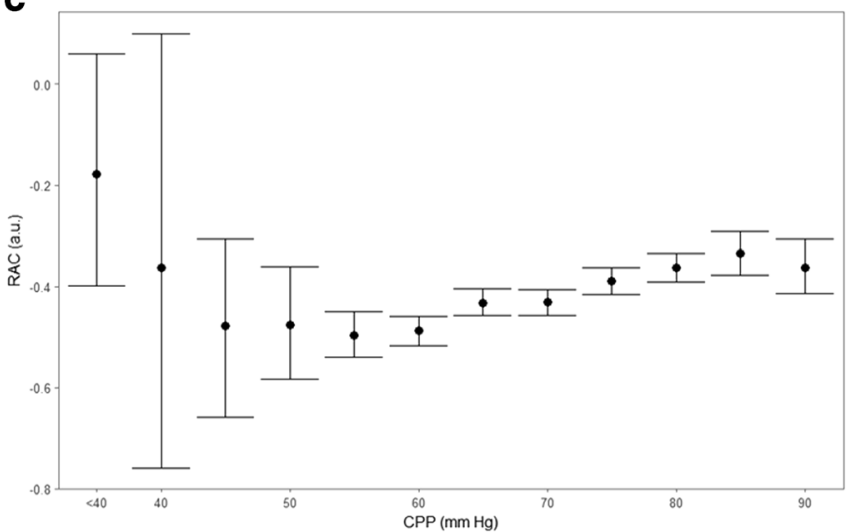

Fig. 3 Population wide error bar plots - ICP-derived indices. AMP, pulse amplitude of ICP; a.u., arbitrary units; CPP, cerebral perfusion pressure; ICP, intracranial pressure; MAP, mean arterial pressure; $\mathrm{mmHg}$, millimeters of mercury; PAx, pulse amplitude index (correlation between AMP and MAP); PRx, pressure reactivity index (correlation between ICP and MAP); RAC, correlation between AMP and CPP. a PRx vs. CPP. b PAx vs. CPP. c RAC vs. CPP. Note: the lack of distinct

recordings reported within this analysis allowed for a single spot assessment of CPPopt using robotic TCD. Thus, in its current state, this allows for a limited "spot" check of a patient's individualized CPPopt, unlike current invasively derived CPPopt which provides a continuous updating value more applicable for routine clinical monitoring. The goal of this study was not to demonstrate equivalency or superiority of TCD-based CPPopt over existing invasively derived versions, but to display some potential uses of this ever evolving robotic TCD technology. As such, no direct comparison between such CPPopt values is provided in this preliminary pilot analysis, as it would be improper to do so based on the limited data acquired. It must emphasized, that this is just a pilot analysis and requires much further work in larger populations with extended duration TCD recordings, in order to determine the role for TCD-based CPPopt and whether these measures relate to clinically relevant outcomes in adult TBI. Furthermore, comparison of TCD-based CPPopt and ICPderived CPPopt needs to occur in a large cohort of patients parabolic relationship in the PRx plot likely stems for the low mean ICP (i.e., $\sim 11 \mathrm{mmHg}$ ) for the population over the course of the 3- to 4-h recordings. PAx and RAC display superior parabolic relationships with CPP likely secondary to the slow-wave fluctuation between AMP and $\mathrm{MAP} / \mathrm{CPP}$ being easier to assess than that of ICP and MAP in the setting of persistently low ICP values

with long TCD recordings, in order to assess if the temporal profiles of these two CPPopt measures are the same, or at least related over time.

Another important aspect is that CPPopt is not a validated CPP target in TBI, as of yet. The concept of CPPopt directed therapy in moderate/severe TBI has not been validated in large prospective randomized control trials. However, numerous retrospective studies have been published assessing the relationship between CPP values outside of the CPPopt range, and global outcome [1, 19, 24]. Having CPP below CPPopt has been documented to be associated with poor global outcome, mortality, and morbidity, in adult TBI [14, 19, 24]. One may also be concerned that targeting CPPopt will lead to a "drift" in CPP targets to higher levels, above and beyond the currently recommended BTF guidelines [9]. However, the association with CPP values above CPPopt is quite unclear. One retrospective study demonstrated a potential association with increase severe disability, though this has not been replicated [1]. Other studies have failed to produce statistically 
a

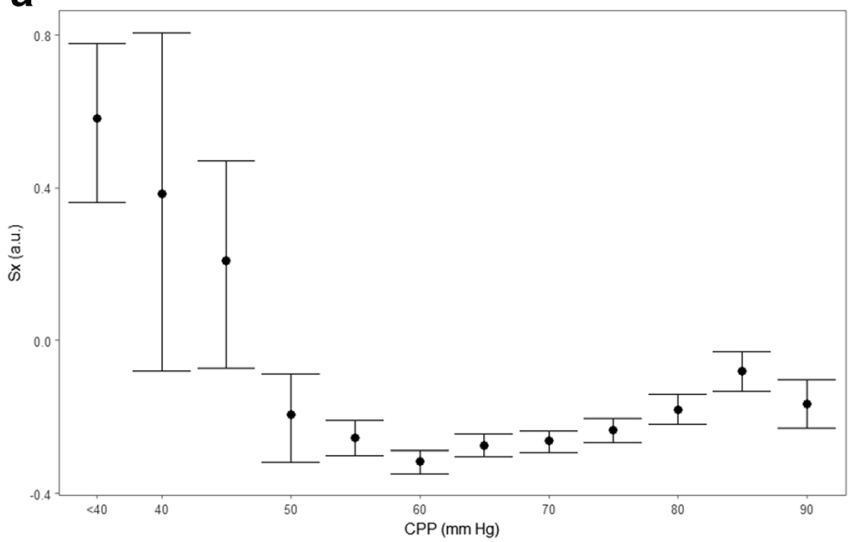

C

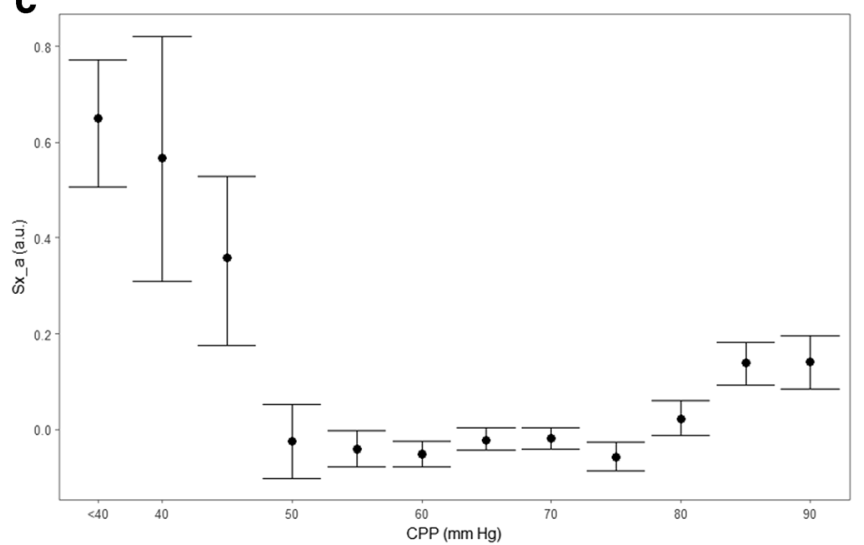

Fig. 4 Population wide error bar plots-TCD-based indices. a.u., arbitrary units; CPP, cerebral perfusion pressure; FVm, mean flow velocity; FVs, systolic flow velocity; ICP, intracranial pressure; MAP, mean arterial pressure; $\mathrm{mmHg}$, millimeters of mercury; $\mathrm{Mx}$, mean flow index (correlation between FVm and CPP); Mx_a, MAP-based mean

significant associations between CPP above CPPopt and patient global outcome [19]. Furthermore, recent work suggests that CPP values above CPPopt may not even be linked to cardiopulmonary complications, such as acute respiratory distress syndrome (ARDS) [25, 29], which has long been the concern of having CPP values above the defined BTF guidelines. All of this uncertainty has led to the phase II randomized control trial of CPPopt versus BTF-based CPP therapy in adult TBI. It will not be until the completion of the planned phase III trial that we will be able to potentially comment on the above outlined uncertainties with CPPopt, and clarify the role of CPPopt therapy in adult TBI.

Given the recorded population consisted of only a small pilot group during the trial period for this device, the ability to extrapolate the results of this study to other populations is limited. This small population is secondary to current technology limitations (as described in the results) and ongoing user presence during recordings. Thus, with this technology, TCD becomes less involved, however, it is still somewhat labor intensive compared to other monitoring devices employed in critically ill TBI patients. b

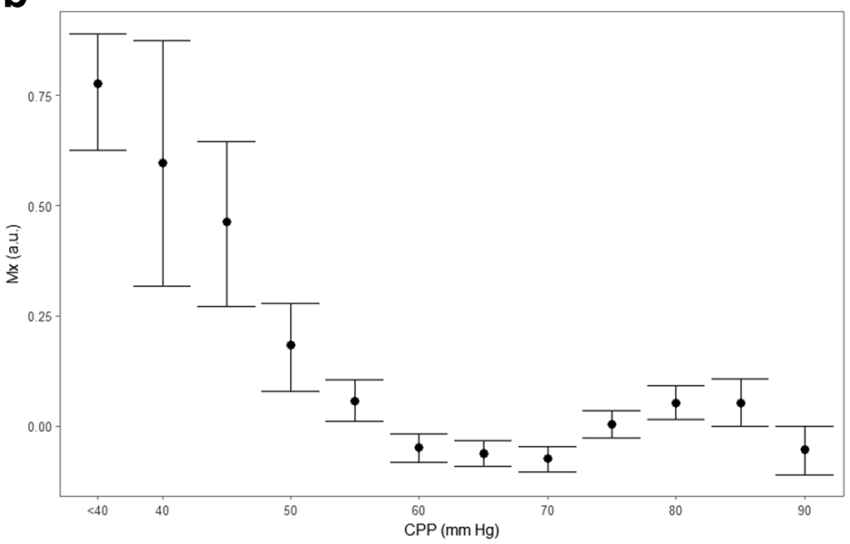

d

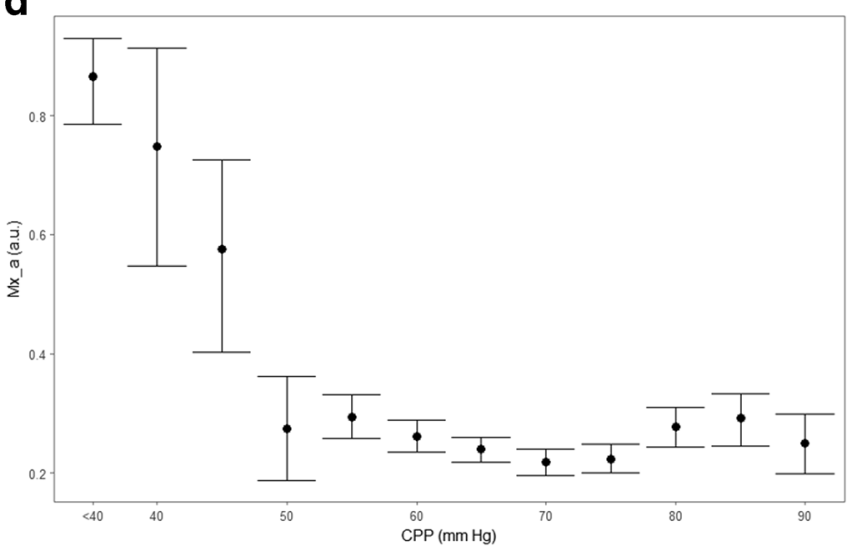

flow index (correlation between FVm and MAP); Sx, systolic flow index (correlation between FVs and CPP); Sx_a, MAP-based systolic flow index (correlation between FVs and MAP). a Sx vs. CPP. b Mx vs. CPP. c: Sx_a vs. CPP. d Mx_a vs. CPP

One may question the use of TCD in the presence of existing ICP monitoring, particularly when it comes to assessing autoregulation/vascular reactivity. However, ICP, as used in PRx calculations, has some conceptual problems when assessing cerebrovascular reactivity. First, ICP is considered a surrogate for changes in pulsatile cerebral blood volume (CBV) and relies on the pressure-volume relationship. TCD is a close direct measure to cerebral blood flow (CBF) and likely provides more useful information for assessment of autoregulation. Second, PRx does not measure autoregulation directly; it is an index of vasodilation/constriction in response to $\mathrm{CPP}$ changes, whereas TCD flow velocity reflects changes in CBF, which is what we are really interested in. Finally, ICP is a regional measure that is approximated to global compartmental reading. With TCD, one can obtain territory specific information and assess hemispheric asymmetry.

The concept of TCD-based CPPopt may be of question as well, since CPP currently requires ICP to obtain. However, efforts are ongoing for non-invasive TCD-based methods of CPP measurement $[7,8,15,21,26,27]$. With the acquisition of extended duration uninterrupted robotic TCD recordings, the 
potential to have a more continuous TCD-based CPP measure is feasible, though further research is required. Combining TCD measured CPP with non-invasive TCD cerebrovascular reactivity indices, such as $\mathrm{Sx} \_\mathrm{a}$ or $\mathrm{Mx} \_\mathrm{a}$ (acquired using TCD in conjunction with non-invasive continuous $\mathrm{ABP}$ ), one could in theory obtain CPPopt through an entirely non-invasive means. Again, much further work in this area is required. However, as robotic TCD technology continues to improve, we will see longer duration of uninterrupted recordings, and hopefully a move to less invasive monitoring in TBI.

\section{Conclusions}

With the application of robotic TCD technology, it is possible to obtain extended duration recordings in critically ill TBI patients, allowing for the approximation of CPPopt using TCDbased cerebrovascular reactivity indices. As robotic TCD technology continues to advance, further long-term recording is becoming possible, with minimal user input, allowing for inclusion of continuous, uninterrupted, TCD monitoring into the standard set of neuromonitoring modalities in TBI patients.

Acknowledgments This work was made possible through salary support through the Cambridge Commonwealth Trust Scholarship, the Royal College of Surgeons of Canada - Harry S. Morton Traveling Fellowship in Surgery and the University of Manitoba Clinician Investigator Program.

Statement of authorship FAZ was responsible for concept, data collection, data analysis, statistical methodology, and manuscript composition. $\mathrm{MC}$ and PS were responsible for concept, manuscript writing, and editing.

\section{Compliance with ethical standards}

Disclosures FAZ has received salary support for dedicated research time, during which this project was partially completed. Such salary support came from the Cambridge Commonwealth Trust Scholarship, the Royal College of Surgeons of Canada - Harry S. Morton Traveling Fellowship in Surgery and the University of Manitoba Clinician Investigator Program. PS has financial interest in a part of licensing fee for ICM+ software (Cambridge Enterprise Ltd., UK).

$\mathrm{MC}$ has financial interest in a part of licensing fee for ICM+ software (Cambridge Enterprise Ltd., UK).

Conflict of interest The authors that they have no conflict of interest.

Ethical approval All procedures performed in studies involving human participants were in accordance with the ethical standards of the institutional and/or national research committee (name of institute/committee) and with the 1964 Helsinki declaration and its later amendments or comparable ethical standards. All data related to patient admission demographics and high frequency digital signals from monitoring devices were collected in an entirely anonymous format, negating the need for formal consent, as in accordance with institutional research committee policies.

Informed consent For this type of study, formal informed consent was not required.
Open Access This article is distributed under the terms of the Creative Commons Attribution 4.0 International License (http:// creativecommons.org/licenses/by/4.0/), which permits unrestricted use, distribution, and reproduction in any medium, provided you give appropriate credit to the original author(s) and the source, provide a link to the Creative Commons license, and indicate if changes were made.

\section{References}

1. Aries MJH, Czosnyka M, Budohoski KP et al (2012) Continuous determination of optimal cerebral perfusion pressure in traumatic brain injury. Crit Care Med 40(8):2456-2463

2. Aries MJH, Wesselink R, Elting JWJ, Donnelly J, Czosnyka M, Ercole A, Maurits NM, Smielewski P (2016) Enhanced visualization of optimal cerebral perfusion pressure over time to support clinical decision making. Crit Care Med 44(10):e996-e999

3. Blanco P (2016) Transcranial color-coded duplex sonography: another option besides the blind method. J Ultrasound Med 35(3): 669-671

4. Brady KM, Lee JK, Kibler KK, Easley RB, Koehler RC, Shaffner DH (2008) Continuous measurement of autoregulation by spontaneous fluctuations in cerebral perfusion pressure: comparison of 3 methods. Stroke 39(9):2531-2537

5. British Medical Ultrasound Society (2012) The British Medical Ultrasound Society: guidelines for the safe use of diagnostic ultrasound equipment

6. Budohoski KP, Reinhard M, Aries MJH, Czosnyka Z, Smielewski P, Pickard JD, Kirkpatrick PJ, Czosnyka M (2012) Monitoring cerebral autoregulation after head injury. Which component of transcranial Doppler flow velocity is optimal? Neurocrit Care 17(2): 211-218

7. Calviello LA, de Riva N, Donnelly J, Czosnyka M, Smielewski P, Menon DK, Zeiler FA (2017) Relationship between brain pulsatility and cerebral perfusion pressure: replicated validation using different drivers of CPP change. Neurocrit Care 27(3):392-400

8. Cardim D, Robba C, Bohdanowicz M, Donnelly J, Cabella B, Liu X, Cabeleira M, Smielewski P, Schmidt B, Czosnyka M (2016) Non-invasive monitoring of intracranial pressure using transcranial Doppler ultrasonography: is it possible? Neurocrit Care 25(3):473-491

9. Carney N, Totten AM, O'Reilly C et al (2017) Guidelines for the management of severe traumatic brain injury, fourth edition. Neurosurgery 80(1):6-15

10. Czosnyka M, Miller C, Participants in the International Multidisciplinary Consensus Conference on Multimodality Monitoring (2014) Monitoring of cerebral autoregulation. Neurocrit Care 21 Suppl 2:S95-102

11. Czosnyka M, Smielewski P, Kirkpatrick P, Laing RJ, Menon D, Pickard JD (1997) Continuous assessment of the cerebral vasomotor reactivity in head injury. Neurosurgery 41(1):11-17 discussion 17-19

12. ter Haar G (2012) The safe use of ultrasound in medical diagnosis, 3rd edn. The British Institute of Radiology, London

13. Harris GR, Church CC, Dalecki D, Ziskin MC, Bagley JE, American Institute of Ultrasound in Medicine, Health Canada, British Medical Ultrasound Society (2016) Comparison of thermal safety practice guidelines for diagnostic ultrasound exposures. Ultrasound Med Biol 42(2):345-357

14. Howells T, Smielewski P, Donnelly J, Czosnyka M, Hutchinson PJA, Menon DK, Enblad P, Aries MJH (2018) Optimal cerebral perfusion pressure in centers with different treatment protocols. Crit Care Med 46(3):e235-e241 
15. Khan MN, Shallwani H, Khan MU, Shamim MS (2017) Noninvasive monitoring intracranial pressure - a review of available modalities. Surg Neurol Int 8:51

16. Le Roux P, Menon DK, Citerio G et al (2014) The international multidisciplinary consensus conference on multimodality monitoring in neurocritical care: evidentiary tables: a statement for healthcare professionals from the Neurocritical Care Society and the European Society of Intensive Care Medicine. Neurocrit Care 21(Suppl 2):S297-S361

17. Liu X, Maurits NM, Aries MJH et al (2017) Monitoring of optimal cerebral perfusion pressure in traumatic brain injured patients using a multi-window weighting algorithm. J Neurotrauma 34(22):3081-3088

18. Mackinnon AD, Aaslid R, Markus HS (2004) Long-term ambulatory monitoring for cerebral emboli using transcranial Doppler ultrasound. Stroke 35(1):73-78

19. Needham E, McFadyen C, Newcombe V, Synnot AJ, Czosnyka M, Menon D (2017) Cerebral perfusion pressure targets individualized to pressure-reactivity index in moderate to severe traumatic brain injury: a systematic review. J Neurotrauma 34(5):963-970

20. Postert T, Federlein J, Przuntek H, Büttner T (1998) Comparison of transcranial power Doppler and contrast-enhanced color-coded sonography in the identification of intracranial arteries. J Ultrasound Med 17(2):91-96

21. Robba C, Cardim D, Sekhon M, Budohoski K, Czosnyka M (2017) Transcranial Doppler: a stethoscope for the brain-neurocritical care use. J Neurosci Res. https://doi.org/10.1002/jnr.24148

22. Sorrentino E, Budohoski KP, Kasprowicz M, Smielewski P, Matta B, Pickard JD, Czosnyka M (2011) Critical thresholds for transcranial Doppler indices of cerebral autoregulation in traumatic brain injury. Neurocrit Care 14(2):188-193

23. Sorrentino E, Diedler J, Kasprowicz M et al (2012) Critical thresholds for cerebrovascular reactivity after traumatic brain injury. Neurocrit Care 16(2):258-266

24. Steiner LA, Czosnyka M, Piechnik SK, Smielewski P, Chatfield D, Menon DK, Pickard JD (2002) Continuous monitoring of cerebrovascular pressure reactivity allows determination of optimal cerebral perfusion pressure in patients with traumatic brain injury. Crit Care Med 30(4):733-738

25. Thiara S, Griesdale DE, Henderson WR, Sekhon MS (2018) Effect of cerebral perfusion pressure on acute respiratory distress syndrome. Can J Neurol Sci:1-7

26. Varsos GV, Kasprowicz M, Smielewski P, Czosnyka M (2014) Model-based indices describing cerebrovascular dynamics. Neurocrit Care 20(1):142-157

27. Varsos GV, Kolias AG, Smielewski P, Brady KM, Varsos VG, Hutchinson PJ, Pickard JD, Czosnyka M (2015) A noninvasive estimation of cerebral perfusion pressure using critical closing pressure. J Neurosurg 123(3):638-648
28. Watt BP (2012) Design and development of a novel transcranial Doppler headset for assessment of cerebral blood flow. Thesis, University of Nebraska

29. Zeiler F (2018) Cerebral perfusion pressure targets in traumatic brain injury: the "fuzzy" spots above optimal cerebral perfusion pressure. Can J Neurol Sci In Press

30. Zeiler FA, Cardim D, Donnelly J, Menon DK, Czosnyka M, Smielewski P (2018) Transcranial Doppler systolic flow index and ICP-derived cerebrovascular reactivity indices in traumatic brain injury. J Neurotrauma 35(2):314-322

31. Zeiler FA, Donnelly J, Calviello L, Menon DK, Smielewski P, Czosnyka M (2017) Pressure autoregulation measurement techniques in adult traumatic brain injury, part I: a scoping review of intermittent/semi-intermittent methods. J Neurotrauma 34(23): 3207-3223

32. Zeiler FA, Donnelly J, Calviello L, Smielewski P, Menon DK, Czosnyka M (2017) Pressure autoregulation measurement techniques in adult traumatic brain injury, part II: a scoping review of continuous methods. J Neurotrauma 34(23):3224-3237

33. Zeiler FA, Smieleweski P, Donnelly J, Czosnyka M, Menon D, Ercole A (2018) Estimating pressure reactivity index using noninvasive Doppler based systolic flow index. J Neurotrauma. https://doi.org/10.1089/neu.2017.5596

34. Zeiler FA, Smielewski P (2018) Application of robotic transcranial Doppler for extended duration recording in moderate/severe traumatic brain injury: first experiences. Crit Ultrasound J 10(1):16

\section{Comments}

In the management of patients with traumatic brain injury, the concept of optimal cerebral perfusion pressure has emerged as the perfusion pressure associated with the lowest change in cerebral blood flow and, accordingly, intracranial pressure with a certain change in perfusion pressure. The present study compares transcranial Doppler ultrasoundbased measurement of cerebral blood flow velocity with 'conventional' intracranial pressure measurement to calculate the pressure reactivity in a selected cohort of patients. TCD was performed continuously using a robotic device, minimizing the need for manual adjustment. Even though important issues remain to be clarified, including safety considerations for the different technologies, individually tailored and targeted management appears to represent an important next step to optimize the outcome for neurocritically ill patients.

Kirsten Moeller

Denmark 